\title{
A construction for the hat problem on a directed graph
}

\author{
Rani Hod* \\ School of Computer Science \\ Raymond and Beverly Sackler Faculty of Exact Sciences \\ Tel Aviv University, Tel Aviv, Israel \\ rani.hod@cs.tau.ac.il \\ Marcin Krzywkowski \\ Faculty of Electronics, Telecommunications and Informatics \\ Gdańsk University of Technology \\ Narutowicza 11/12, 80-233 Gdańsk, Poland \\ marcin.krzywkowski@gmail.com
}

Submitted: Aug 29, 2011; Accepted: Jan 18, 2012; Published: Jan 27, 2012

Mathematics Subject Classification: 05C20, 05C69, 91A12, 91A43

\begin{abstract}
A team of $n$ players plays the following game. After a strategy session, each player is randomly fitted with a blue or red hat. Then, without further communication, everybody can try to guess simultaneously his own hat color by looking at the hat colors of the other players. Visibility is defined by a directed graph; that is, vertices correspond to players, and a player can see each player to whom he is connected by an arc. The team wins if at least one player guesses his hat color correctly, and no one guesses his hat color wrong; otherwise the team loses. The team aims to maximize the probability of a win, and this maximum is called the hat number of the graph.

Previous works focused on the hat problem on complete graphs and on undirected graphs. Some cases were solved, e.g., complete graphs of certain orders, trees, cycles, and bipartite graphs. These led Uriel Feige to conjecture that the hat number of any graph is equal to the hat number of its maximum clique.

We show that the conjecture does not hold for directed graphs. Moreover, for every value of the maximum clique size, we provide a tight characterization of the range of possible values of the hat number. We construct families of directed graphs with a fixed clique number the hat number of which is asymptotically optimal. We also determine the hat number of tournaments to be one half.
\end{abstract}

Keywords: hat problem, directed graph, digraph, skeleton, clique number.

\footnotetext{
*Research supported by an ERC advanced grant.
} 


\section{Introduction}

In the hat problem, a team of $n$ players enters a room and a blue or red hat is randomly and independently placed on the head of each player. Each player can see the hats of all of the other players but not his own. No communication of any sort is allowed, except for an initial strategy session before the game begins. Once they have had a chance to look at the other hats, each player must simultaneously guess the color of his own hat or pass. The team wins if at least one player guesses his hat color correctly and no one guesses his hat color wrong; otherwise the team loses. The aim is to maximize the probability of a win.

The hat problem with seven players, called the "seven prisoners puzzle", was formulated by Todd Ebert in his Ph.D. Thesis [6]. It is often posed as a puzzle (e.g., in the Berkeley Riddles [2]) and was also the subject of articles in popular media [3, 20, 21].

The hat problem with $q \geq 2$ possible colors was investigated in [19]. Noga Alon [1] proved that the $q$-ary hat number of the complete graph tends to one as the graph grows.

Many other variations of the problem exist (for a comprehensive list, see [15]), among them a random but non-uniform hat color distribution [10], an adversarial allocation of hat from a pool known by the players [9], a variation in which passing is not allowed [4], a variation in which players do not have to guess their hat colors simultaneously [11], and many more.

The hat problem can be considered on a graph, where vertices correspond to players, and a player can see each player to whom he is connected by an edge. We seek to determine the hat number of the graph, that is, the maximum chance of success for the hat problem on it. This variation of the hat problem was first considered in [12], and further studied for example in $[8,13,14,16-18]$.

Note that the hat problem on the complete graph is equivalent to the original hat problem. This case was solved for $2^{k}-1$ players in [7] and for $2^{k}$ players in [5]. In [19] it was shown that a strategy for $n$ players in the complete graph is equivalent to a covering code of radius 1 in the Hamming cube.

The hat problem was solved for trees [12], cycles [8, 13, 14, 18], bipartite graphs [8], perfect graphs [8], and planar graphs containing a triangle [8]. Feige [8] conjectured that for any graph the hat number is equal to the hat number of its maximum clique. He proved this for graphs with clique number $2^{k}-1$. Thus triangle-free graphs are the simplest remaining open case.

We consider the hat problem on directed graphs. Under an appropriate definition of the clique number for directed graphs, we provide a tight characterization of the range of possible values of the hat number, for every size of the maximum clique. We construct families of directed graphs with a fixed clique number the hat number of which is asymptotically optimal. We also determine the hat number of tournaments to be one half. 


\section{Preliminaries}

For a graph $G$, the set of vertices and the set of edges we denote by $V(G)$ and $E(G)$, respectively. If $H$ is a subgraph of $G$, then we write $H \subseteq G$. The degree of vertex $v$, that is, the number of its neighbors, we denote by $d_{G}(v)$.

Let $f: X \rightarrow Y$ be a function. If for every $x \in X$ we have $f(x)=y$, then we write $f \equiv y$.

Let $V(G)=\left\{v_{1}, v_{2}, \ldots, v_{n}\right\}$. By $S c=\{1,2\}$ we denote the set of colors, where 1 corresponds to blue and 2 corresponds to red.

By a case for a graph $G$ we mean a function $c: V(G) \rightarrow\{1,2\}$, where $c\left(v_{i}\right)$ means color of vertex $v_{i}$. The set of all cases for the graph $G$ we denote by $C(G)$, of course $|C(G)|=2^{|V(G)|}$.

By a situation of a vertex $v_{i}$ we mean a function $s_{i}: V(G) \rightarrow S c \cup\{0\}=\{0,1,2\}$, where $s_{i}\left(v_{j}\right) \in S c$ if $v_{i}$ and $v_{j}$ are adjacent, and 0 otherwise. The set of all possible situations of $v_{i}$ in the graph $G$ we denote by $S t_{i}(G)$, of course $\left|S t_{i}(G)\right|=2^{d_{G}\left(v_{i}\right)}$.

By a guessing instruction of a vertex $v_{i} \in V(G)$ we mean a function $g_{i}: S t_{i}(G) \rightarrow S c$ $\cup\{0\}=\{0,1,2\}$, which for a given situation gives the color $v_{i}$ guesses it is, or 0 if $v_{i}$ passes. Thus a guessing instruction is a rule determining the behavior of a vertex in every situation. We say that $v_{i}$ never guesses its color if $v_{i}$ passes in every situation, that is, $g_{i} \equiv 0$. We say that $v_{i}$ always guesses its color if $v_{i}$ guesses its color in every situation, that is, for every $s_{i} \in S t_{i}(G)$ we have $g_{i}\left(s_{i}\right) \in\{1,2\}\left(g_{i}\left(s_{i}\right) \neq 0\right.$, equivalently).

Let $c$ be a case, and let $s_{i}$ be the situation (of vertex $v_{i}$ ) corresponding to that case. The guess of $v_{i}$ in the case $c$ is correct (wrong, respectively) if $g_{i}\left(s_{i}\right)=c\left(v_{i}\right)\left(0 \neq g_{i}\left(s_{i}\right) \neq c\left(v_{i}\right)\right.$, respectively). By result of the case $c$ we mean a win if at least one vertex guesses its color correctly, and no vertex guesses its color wrong, that is, $g_{i}\left(s_{i}\right)=c\left(v_{i}\right)$ (for some $i$ ) and there is no $j$ such that $0 \neq g_{j}\left(s_{j}\right) \neq c\left(v_{j}\right)$. Otherwise the result of the case $c$ is a loss.

By a strategy for the graph $G$ we mean a sequence $\left(g_{1}, g_{2}, \ldots, g_{n}\right)$, where $g_{i}$ is the guessing instruction of vertex $v_{i}$. The family of all strategies for a graph $G$ we denote by $\mathcal{F}(G)$.

If $S \in \mathcal{F}(G)$, then the set of cases for the graph $G$ for which the team wins (loses, respectively) using the strategy $S$ we denote by $W(S)(L(S)$, respectively). The set of cases for which the team loses, and some vertex guesses its color we denote by $\operatorname{Ls}(S)$. By the chance of success of the strategy $S$ we mean the number $p(S)=|W(S)| /|C(G)|$. By the hat number of the graph $G$ we mean the number $h(G)=\max \{p(S): S \in \mathcal{F}(G)\}$. We say that a strategy $S$ is optimal for the graph $G$ if $p(S)=h(G)$. The family of all optimal strategies for the graph $G$ we denote by $\mathcal{F}^{0}(G)$.

By solving the hat problem on a graph $G$ we mean finding the number $h(G)$.

For a directed graph (digraph) $D$, the set of vertices and the set of arcs we denote by $V(D)$ and $A(D)$, respectively.

By the skeleton of a digraph $D$, denoted by skel $(D)$, we mean the undirected graph on the vertex set $V$ in which $x$ and $y$ are adjacent if both arcs between them belong to the set $A$, that is, if they form a directed 2-cycle in $D$.

By the clique number of a digraph $D$ we mean the clique number of its skeleton; 
that is, $\omega(D)=\omega(\operatorname{skel}(D))$.

The transpose of a digraph $D=(V, A)$ is the digraph $D^{t}=\left(V, A^{t}\right)$, where $A^{t}$ $=\{(x, y):(y, x) \in A\}$.

Slightly abusing notation, we identify a digraph $D$ with its (undirected) skeleton in the case that $D=D^{t}$, that is, if all arcs of $D$ have anti-parallel counterparts.

We can also consider the hat problem on directed graphs. If there is an arc from $u$ to $v$, then the vertex $u$ can see the vertex $v$. All concepts we define similarly as when considering the hat problem on undirected graphs treated for example in [8, 12]. We now cite four propositions that generalize to digraphs with little or no change.

Proposition 1 For every two digraphs $D$ and $E$ such that $E \subseteq D$ we have $h(E) \leq h(D)$.

Proposition 2 For every digraph $D$ we have $h(D) \geq 1 / 2$.

Proposition 3 Let $D$ be a digraph and let $v$ be a vertex of $D$. If $S \in \mathcal{F}^{0}(D)$ is a strategy such that $v$ always guesses its color, then $h(D)=1 / 2$.

Proposition 4 Let $D$ be a digraph and let $v$ be a vertex of $D$. If $S \in \mathcal{F}^{0}(D)$ is a strategy such that $v$ never guesses its color, then $h(D)=h(D-v)$.

We have the following corollary from Propositions 2, 3, and 4 .

Proposition 5 Let $D$ be a digraph and let $v$ be a vertex of $D$. If $v$ has no outgoing arcs, then $h(D)=h(D-v)$.

\section{Constructions}

For an undirected graph $G$, it is known that if $G$ contains a triangle, then $h(G) \geq 3 / 4$, and in [8] it is conjectured that if $G$ is triangle-free, then $h(G)=1 / 2$. Do directed graphs introduce anything in between? The answer is yes.

Let us consider the hat problem on the digraph $D_{1}$ given in Figure 1.

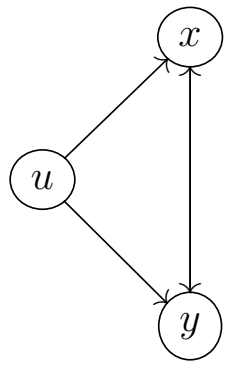

Figure 1: The digraph $D_{1}$

Fact $6 h\left(D_{1}\right)=5 / 8$. 
We omit the proof of this fact in favor of extending $D_{1}$ to a construction of a family $\left\{D_{n}\right\}_{n=0}^{\infty}$ of semi-complete digraphs that asymptotically achieve the hat number $2 / 3$, with the property that $\omega\left(D_{n}\right)=2$. The skeleton of $D_{n}$ is a matching of size $n$ plus an isolated vertex. For short, we write $\operatorname{skel}\left(D_{n}\right)=n K_{2} \cup K_{1}$.

Definition 7 Given two disjoint digraphs $C$ and $D$, we define the directed union of $C$ and $D$, denoted by $C \rightarrow D$, to be the union of these two digraphs with the additional arcs from all vertices of $C$ to all vertices of $D$. Note that this operator is associative, that is, $C \rightarrow(D \rightarrow E)=(C \rightarrow D) \rightarrow E$, for any three digraphs $C, D$ and $E$. Thus the notation $C \rightarrow D \rightarrow E$ is unambiguous. The directed union of $n$ disjoint copies of a digraph $D$, that is $\underbrace{D \rightarrow D \rightarrow \ldots \rightarrow D}_{n}$, we denote by $D^{\rightarrow n}$.

Expressed in the terms of the directed union, $D_{1}=K_{1} \rightarrow K_{2}$. We extend this to a family of digraphs by defining $D_{n}=K_{1} \rightarrow K_{2}^{\rightarrow n}$. Note that the family $\left\{D_{n}\right\}_{n=0}^{\infty}$ satisfies the recurrence relation $D_{n+1}=D_{n} \rightarrow K_{2}$.

In Figure 2 we give examples of $D_{n}$ for $n=2, n=3$, and a general $n$.

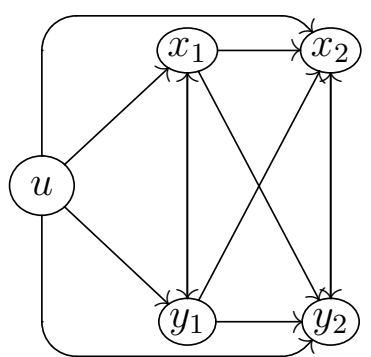

(a) $n=2$

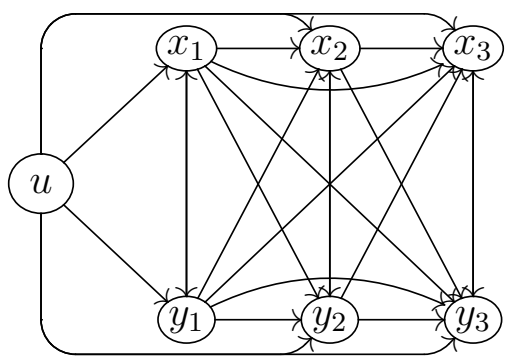

(b) $n=3$

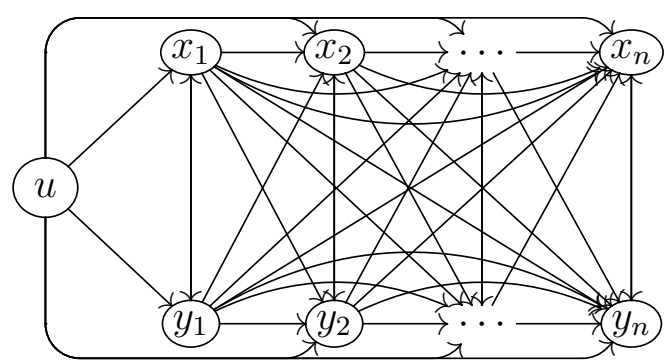

(c) General $n$

Figure 2: The directed, semi-complete graphs $D_{2}, D_{3}$, and $D_{n}$. All vertical arcs have anti-parallel counterparts. The remaining arcs are rightwards

We proceed to compute the hat number of the digraphs of the family $\left\{D_{n}\right\}_{n=0}^{\infty}$. First we prove an upper bound.

Lemma 8 For every digraph $D$ we have $h\left(D \rightarrow K_{2}\right) \leq \max \{h(D), 1 / 2+h(D) / 4\}$.

Proof. Let $S$ be an optimal strategy for $D \rightarrow K_{2}$. The vertices of the $K_{2}$ we denote by $x$ and $y$. If one of them, say $x$, never guesses its color, then using Propositions 4 and 5 we get $h\left(D \rightarrow K_{2}\right)=h\left(D \rightarrow K_{2}-x\right)=h\left(D \rightarrow K_{1}\right)=h(D)$. Now assume that each one of the vertices $x$ and $y$ guesses its color. If $x$ or $y$ always guesses its color, then by Proposition 3 we have $h\left(D \rightarrow K_{2}\right)=1 / 2$. Now assume that neither $x$ nor $y$ always guesses its color. This implies that each one of them guesses its color in one of the two situations as every one of them has just one outgoing arc. Hence, with probability at least $1 / 4$ at least one of the vertices $x$ and $y$ is wrong. The chance of the success of the strategy $S$ benefits from the behavior of the vertices of $D$ only when both $x$ and $y$ pass, and this happens exactly 
with probability $1 / 4$ since they see different vertices (that is, each other). This implies that $p(S)=1 / 2+h(D) / 4$. Now we get $h\left(D \rightarrow K_{2}\right)=p(S) \leq \max \{h(D), 1 / 2+h(D) / 4\}$.

Now we prove a lower bound.

Lemma 9 For every digraph $D$ we have $h\left(D \rightarrow K_{2}\right) \geq 1 / 2+h(D) / 4$.

Proof. Let $S$ be an optimal strategy for the digraph $D$. The vertices of the $K_{2}$ we denote by $x$ and $y$. Let $S^{\prime}$ be a strategy for $D \rightarrow K_{2}$ as follows. If $y$ is blue, then $x$ guesses it is also blue; otherwise it passes. If $x$ is red, then $y$ guesses it is also red; otherwise it passes. If $x$ is blue and $y$ is red, then the vertices of $D$ behave as in the strategy $S$, otherwise they pass. Let us observe that if $x$ and $y$ have the same color, then the team wins. If $x$ is red and $y$ is blue, then the team loses. If $x$ is blue and $y$ is red, then the team wins with probability $p(S)$. Therefore $p\left(S^{\prime}\right)=1 / 2+p(S) / 4=1 / 2+h(D) / 4$. Consequently, $h\left(D \rightarrow K_{2}\right) \geq p\left(S^{\prime}\right)=1 / 2+h(D) / 4$.

Now we prove a lower bound for a more general setting.

Lemma 10 For every positive integer $m$ there exists $c \geq 1 / 2$ such that for any digraph $D$ we have $h\left(D \rightarrow K_{m}\right) \geq \mathrm{cm} /(m+1)+(1-c) \cdot h(D)$. Moreover, if $m=2$, then $c=3 / 4$ satisfies the inequality.

Proof. Let $S$ be an optimal strategy for the digraph $D$. The vertices of $K_{m}$ we denote by $x_{1}, x_{2}, \ldots, x_{m}$. Let $C \subset\{\text { blue, red }\}^{m}$ be a code of distance 3 , and consider the packing of stars $K_{1, m}$ in the hypercube graph $H_{m}$ formed by selecting balls of radius one around each codeword. Let $\mathcal{A}$ mean the event that the case of $x_{1}, x_{2}, \ldots, x_{m}$ is covered by the packing. Now let $S^{\prime}$ be a strategy for $D \rightarrow K_{m}$ as follows. All vertices of $D$ pass if $\mathcal{A}$ occurred, otherwise they behave according to $S$. The vertices of $K_{m}$ behave as follows. If $x_{i}$ is in a situation consistent with some codeword, then it guesses the color that disagrees with it; otherwise it passes. When $\mathcal{A}$ occurs, either $m$ vertices guess their colors wrong, or exactly one vertex guesses its color and the guess is correct; then the team wins with probability $m /(m+1)$. Let $c=p(\mathcal{A})$. We get $p\left(S^{\prime}\right)=p(\mathcal{A}) \cdot m /(m+1)+(1-p(\mathcal{A})) \cdot p(S)$ $=\mathrm{cm} /(m+1)+(1-c) \cdot h(D)$. Now, the existence of codes of distance 3 , length $m$, and size $\left\lceil 2^{m-1} /(m+1)\right\rceil$ implies that $c \geq 1 / 2$.

We use Lemmas 8 and 9 to calculate the hat number of $D_{n}$.

Proposition 11 For every non-negative integer $n$ we have

$$
h\left(D_{n}\right)=\frac{2}{3}-\frac{1}{6} \cdot \frac{1}{4^{n}} .
$$


Proof. The result we prove by induction on the number $n$. For $n=0$ the result is obviously true as $D_{0}$ is a single vertex and $h\left(D_{0}\right)=1 / 2=2 / 3-1 / 6$. Let $n$ be a positive integer, and assume that $h\left(D_{n-1}\right)=2 / 3-4^{1-n} / 6$. Since $h\left(D_{n-1}\right)<2 / 3$, using Lemma 8 we get $h\left(D_{n}\right) \leq \max \left\{h\left(D_{n-1}\right), 1 / 2+h\left(D_{n-1}\right) / 4\right\}=1 / 2+h\left(D_{n-1}\right) / 4$. The lower bound is matched by Lemma 9 .

Corollary 12 For every $\varepsilon>0$ there exists a digraph $D$ satisfying $\omega(D)=2$ such that $h(D)>2 / 3-\varepsilon$.

The previous result can be generalized to an arbitrary clique number $m$.

Theorem 13 For every $\varepsilon>0$ there exists a digraph $D$ satisfying $\omega(D)=m$ such that $h(D)>m /(m+1)-\varepsilon$.

Proof. Let us consider $D=K_{m}^{\rightarrow n}$, where $n=\left\lceil\log _{1-c}(\varepsilon)\right\rceil$ and $c$ is the appropriate constant from Lemma 10. By repeatedly applying the lemma we get $h(D) \geq\left(1-(1-c)^{n}\right)$ $\cdot m /(m+1) \geq(1-\varepsilon) \cdot m /(m+1)>m /(m+1)-\varepsilon$.

A natural question is whether $m /(m+1)$ is the best possible hat number of such digraphs. In the following section we show that indeed this is the best possible, i.e., the chance of success $m /(m+1)$ is asymptotically optimal for digraphs with the clique number $m$.

\section{The upper bound}

Feige [8] proved that for every undirected graph $G$ we have $h(G) \leq \omega(G) /(\omega(G)+1)$. We repeat his proof, refining it a bit to show that the same holds for digraphs.

Proposition 14 For every digraph $D$ we have $h(D) \leq \omega(D) /(\omega(D)+1)$.

Proof. Let $S$ be an optimal strategy for $D$. We define a bipartite graph $B$ whose lefthand side is $L s(S)$, and the right-hand side is $W(S)$. A losing case $l \in L s(S)$ is adjacent to a winning case $w \in W(S)$ if they differ only by one coordinate, which is the color of a vertex $v \in V(D)$ that guesses its color in these cases. Since $v$ cannot see its own hat color, it acts the same in both hat cases $l$ and $w$. Now let us examine the right and left degrees in $B$.

Right degree. Let $w \in W(S)$, and let $v \in V(D)$ be a vertex that guesses its color correctly in $w$. Let $l$ be a case which differs from $w$ only in the color of the vertex $v$. Since $v$ does not distinguish between the cases $w$ and $l$, it makes the same guess in $l$, but now it is incorrect. Therefore $l \in L s(S)$ is a neighbor of $w$ in $B$, and $d(w) \geq 1$. 
Left degree. Let $l \in L s(S)$, and let $w_{1}, w_{2}, \ldots, w_{d_{G}(l)} \in W(S)$ mean the neighbors of $l$ in $B$. Let $v_{i} \in V(D)$ be the vertex whose color differs in the cases $l$ and $w_{i}$, for every $i \in\{1,2, \ldots, d\}$. Suppose that some arc $v_{i} \rightarrow v_{j}$ is not present in $D$. By the definition of $v_{i}$, it makes a correct guess at the case $w_{i}$. It cannot tell $w_{i}$ apart from $l$, and thus it makes the same, now wrong, guess at the case $l$. But then it must make the same incorrect guess at the case $w_{j}$, which only differs from $l$ by the color of $v_{j}$, unseen by $v_{i}$. This contradicts the fact that $w_{j}$ is a winning case. Therefore $\left\{v_{i}\right\}_{i=1}^{d}$ is a clique in $\operatorname{skel}(D)$ and $d=d(l) \leq \omega(\operatorname{skel}(D))=\omega(D)$.

We have shown that the right degree in $B$ is at least one and the left degree in $B$ is at most $\omega(D)$. This implies that $|W(S)| \leq|E(B)| \leq \omega(D)|L s(S)|$, and consequently, $h(D)=p(S)=|W(S)| / 2^{|V(D)|} \leq|W(S)| /(|W(S)|+|L s(S)|) \leq \omega(D) /(\omega(D)+1)$.

Observe that for a digraph $D$, the hat number $h(D)$ is always a rational number whose denominator is a power of two. Therefore $h(D)<\omega(D) /(\omega(D)+1)$ unless $\omega(D)+1$ is a power of two. When $\omega(D)+1=2^{k}$ is a power of two, the upper bound is met by a complete graph $K_{2^{k}-1}$ as $h\left(K_{2^{k}-1}\right)=\left(2^{k}-1\right) / 2^{k}$.

Corollary 15 For every tournament $T$ we have $h(T)=1 / 2$.

Proof. Apply Proposition 14 with $\omega(T)=1$. The lower bound is by Proposition 2 .

\section{Acknowledgments}

The authors thank Noga Alon for useful discussions and comments.

\section{References}

[1] N. Alon, Problems and results in extremal combinatorics - II, Discrete Mathematics 308 (2008), 4460-4472.

[2] Berkeley Riddles, www.ocf.berkeley.edu/ wwu/riddles/hard.shtml.

[3] W. Blum, Denksport für Hutträger, Die Zeit, May 3, 2001.

[4] S. Butler, M. Hajianghayi, R. Kleinberg, and T. Leighton, Hat guessing games, SIAM Journal on Discrete Mathematics 22 (2008), 592-605.

[5] G. Cohen, I. Honkala, S. Litsyn, and A. Lobstein, Covering Codes, North Holland, 1997.

[6] T. Ebert, Applications of recursive operators to randomness and complexity, Ph.D. Thesis, University of California at Santa Barbara, 1998.

[7] T. Ebert, W. Merkle, and H. Vollmer, On the autoreducibility of random sequences, SIAM Journal on Computing 32 (2003), 1542-1569.

[8] U. Feige, On optimal strategies for a hat game on graphs, SIAM Journal on Discrete Mathematics 24 (2010), 782-791. 
[9] U. Feige, You can leave your hat on (if you guess its color), Technical Report MCS0403, Computer Science and Applied Mathematics, The Weizmann Institute of Science, 2004, 10 pp.

[10] W. Guo, S. Kasala, M. Rao, and B. Tucker, The hat problem and some variations, Advances in distribution theory, order statistics, and inference, 459-479, Statistics for Industry and Technology, Birkhäuser Boston, 2007.

[11] M. Krzywkowski, A modified hat problem, Commentationes Mathematicae 50 (2010), 121-126.

[12] M. Krzywkowski, Hat problem on a graph, Mathematica Pannonica 21 (2010), 1-19.

[13] M. Krzywkowski, Hat problem on odd cycles, Houston Journal of Mathematics 37 (2011), 1063-1069.

[14] M. Krzywkowski, Hat problem on the cycle $C_{4}$, International Mathematical Forum 5 (2010), 205-212.

[15] M. Krzywkowski, On the hat problem, its variations, and their applications, Annales Universitatis Paedagogicae Cracoviensis Studia Mathematica 9 (2010), 55-67.

[16] M. Krzywkowski, On the hat problem on a graph, Opuscula Mathematica 32 (2012), 285-296.

[17] M. Krzywkowski, The hat problem on a union of disjoint graphs, Commentationes Mathematicae 51 (2011), 167-176.

[18] M. Krzywkowski, The hat problem on cycles on at least nine vertices, Ars Combinatoria 101 (2011), 3-13.

[19] H. Lenstra and G. Seroussi, On hats and other covers, IEEE International Symposium on Information Theory, Lausanne, 2002.

[20] J. Poulos, Could you solve this \$1 million hat trick?, abcNews, November 29, 2001.

[21] S. Robinson, Why mathematicians now care about their hat color, The New York Times, Science Times Section, page D5, April 10, 2001. 